hep-th/9909135

\title{
Holomorphic Analogs of Topological Gauge Theories*
}

\author{
Alexander D. Popov ${ }^{1}$ \\ Bogoliubov Laboratory of Theoretical Physics \\ JINR, 141980 Dubna, Moscow Region, Russia
}

\begin{abstract}
We introduce a new class of gauge field theories in any complex dimension, based on algebra-valued $(p, q)$-forms on complex $n$-manifolds. These theories are holomorphic analogs of the well-known Chern-Simons and BF topological theories defined on real manifolds. We introduce actions for different special holomorphic BF theories on complex, Kähler and Calabi-Yau manifolds and describe their gauge symmetries. Candidate observables, topological invariants and relations to integrable models are briefly discussed.
\end{abstract}

PACS: 11.15.-q; 11.10.Ef

Keywords: Topological gauge theories; Action functionals; Holomorphic structures

* supported in part by the grant RFBR-99-01-01076

1 E-mail: popov@thsun1.jinr.ru 


\section{Introduction}

Topological field theories [1, 2] were intensively studied, developed and generalized over the last ten years (see e.g. [3] and references therein). Among these theories, of particular interest are non-Abelian Chern-Simons gauge theories [2]. The Chern-Simons (CS) action functional is

$$
S_{\mathrm{CS}}=\int_{X} \operatorname{Tr}\left(A d A+\frac{2}{3} A \wedge A \wedge A\right),
$$

where $X$ is an oriented smooth 3 -manifold, $A$ is a connection 1 -form on a principal $G$-bundle $P$ over $X$, and $G$ is a Lie group. The Euler-Lagrange equations for the action (1.1) are

$$
F_{A}=0
$$

where $F_{A}=d A+A \wedge A$ is the curvature of a connection $A$. Thus, non-Abelian CS theories give a field-theoretic description of flat connections on $G$-bundles over 3-dimensional manifolds $X$, and expectation values of quantum observables for these theories are topological invariants of $X$ [2, 3].

Other interesting topological field theories associated with flat connections are so-called BF theories [⿴囗十 generalizing Abelian models introduced in [5]. These theories can also be considered as a generalization of Chern-Simons theories to arbitrary dimensions. The classical action for a non-Abelian BF theory has the following form:

$$
S_{\mathrm{BF}}=\int_{X} \operatorname{Tr}\left(B \wedge F_{A}\right)
$$

where $X$ is an oriented smooth $n$-manifold, $F_{A}$ is the curvature of a connection 1 -form $A$ on a principal $G$-bundle $P$ over $X$, and $B$ is an $(n-2)$-form with values in the adjoint bundle $\operatorname{ad} P=$ $P \times_{G} \mathfrak{g}$. We consider a semisimple Lie group $G$ and denote by $\mathfrak{g}$ its Lie algebra. The variation of the action (1.3) w.r.t. $B$ gives Eqs.(1.2), and the variation of the action w.r.t. $A$ gives the equations

$$
d_{A} B=0,
$$

where $d_{A}=d+\operatorname{ad} A$ is the covariant differential on $P$. Thus, BF theories describe flat connections $A$ on bundles $P$ over $n$-manifolds $X$ and ad $P$-valued $d_{A}$-closed $(n-2)$-forms $B$.

An interesting class of (topological) gauge models has been introduced by considering the classical limit of the string field theory for open $N=2$ topological strings on Calabi-Yau 3-folds [6, 77. The corresponding action [6] is given by

$$
S_{\mathrm{hCSW}}=\int_{Z} \theta \wedge \operatorname{Tr}\left(A^{0,1} \bar{\partial} A^{0,1}+\frac{2}{3} A^{0,1} \wedge A^{0,1} \wedge A^{0,1}\right),
$$

where $\theta$ is a nowhere vanishing holomorphic $(3,0)$-form on a Calabi-Yau 3-fold $Z$, and $A^{0,1}$ is the $(0,1)$-component of a connection 1 -form $A$ on a principal $G$-bundle $P$ over $Z$. The field equations following from the Chern-Simons-Witten (CSW) action (1.5a) are

$$
F_{A}^{0,2}=\bar{\partial} A^{0,1}+A^{0,1} \wedge A^{0,1}=0
$$

where $\bar{\partial}$ is the $(0,1)$-part of the exterior derivative $d=\partial+\bar{\partial}$, and $F_{A}^{0,2}$ is the $(0,2)$-part of the curvature $F_{A}$ of a $G$-bundle $P$ with a complex structure group $G$. Recently holomorphic CSW theories (1.5) were discussed [8, 9] in the frames of the program on extending the results of Casson, Floer and Jones to Calabi-Yau (CY) threefolds. 
Our discussion can be summarized in the following diagram:

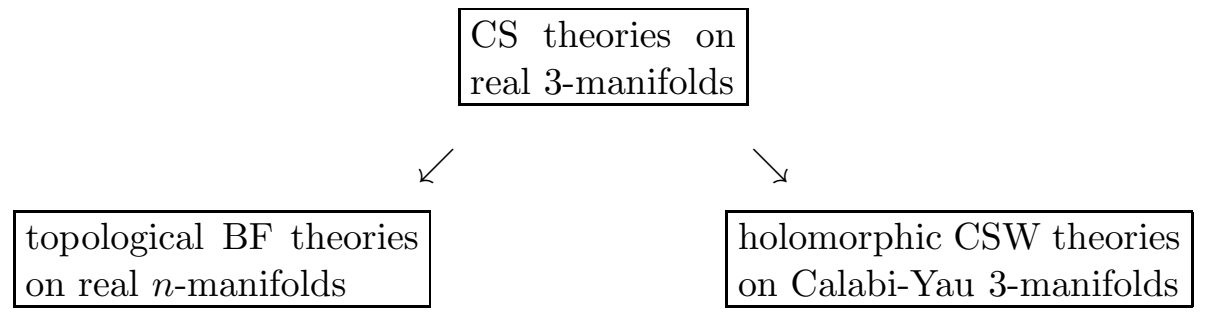

The arrows mean that one theory is a generalization of another one. Notice that holomorphic CSW theories (1.5) are defined only in three complex dimensions by analogy with the fact that CS theories (1.1) with the action linear in derivatives are defined only in three real dimensions. At the same time, it is desirable to have a field theory description of flat $(0,1)$-connections $A^{0,1}$ (holomorphic structures) in arbitrary dimensions. The purpose of our paper is to introduce holomorphic BF theories which generalize both topological BF theories (1.3) and holomorphic CSW theories (1.5) to complex $n$-manifolds and describe holomorphic structures on bundles in any complex dimension. We also introduce three special holomorphic BF theories and describe, in particular, stable holomorphic bundles. So, our goal in this paper is to extend the diagram (1.6) to the diagram

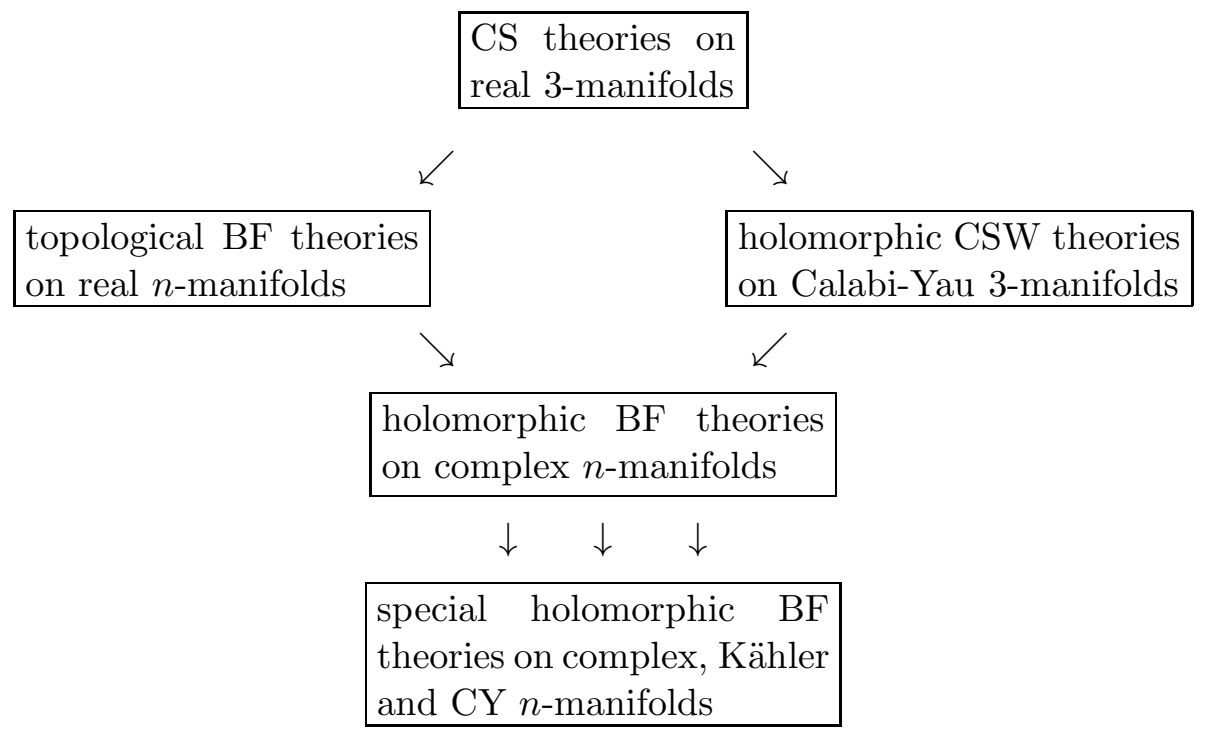

which includes holomorphic analogs not only of Chern-Simons but also of BF theories.

\section{Holomorphic BF theories}

\section{$2.1 \quad$ Non-Abelian models}

Let $Z$ be a complex $n$-manifold, $G$ a (complex) semisimple Lie group, $\mathfrak{g}$ its Lie algebra, $P$ a principal $G$-bundle over $Z$ and $A^{0,1}$ the $(0,1)$-component of a connection 1 -form $A$ on $P$. Components $A^{0,1}$ of connections $A$ will be called $(0,1)$-connections. The curvature $F_{A}$ of a connection $A$ splits into components,

$$
F_{A}=F_{A}^{2,0}+F_{A}^{1,1}+F_{A}^{0,2}
$$


where $F_{A}^{0,2}=\bar{\partial} A^{0,1}+A^{0,1} \wedge A^{0,1}$ is the $(0,2)$-component of the curvature tensor and $\bar{\partial}$ is the $(0,1)$-part of the exterior derivative $d=\partial+\bar{\partial}$. We also split $d_{A}=d+\operatorname{ad} A=\partial_{A}+\bar{\partial}_{A}$.

Let us consider the bundle of groups Int $P=P \times{ }_{G} G(G$ acts on itself by internal automorphisms: $\left.h \mapsto g h g^{-1}, h, g \in G\right)$ associated with $P$. We define the infinite-dimensional group of gauge transformations as the group $\mathcal{G}=\Gamma(Z$, Int $P)$ of global smooth sections of the bundle $\operatorname{Int} P$. We also consider the adjoint bundle $\operatorname{ad} P=P \times_{G} \mathfrak{g}$ of Lie algebras and denote by $\Omega^{p, q}(Z, \operatorname{ad} P)$ the space of smooth $(p, q)$-forms on $Z$ with values in the bundle $\operatorname{ad} P, p, q, \ldots=1, \ldots, n=\operatorname{dim}_{\mathbb{C}} Z$.

For simplicity we shall consider bundles $P$ that are equivalent to the trivial one as smooth bundles (i.e. topologically trivial), $P \simeq Z \times G$. Therefore, we have $\operatorname{Int} P \simeq Z \times G$, ad $P \simeq Z \times \mathfrak{g}$, and $\operatorname{ad} P$-valued $(p, q)$-forms may be considered as $\mathfrak{g}$-valued $(p, q)$-forms on $Z$. The space of such forms is denoted by $\Omega^{p, q}(Z, \mathfrak{g})$. Generalization to the case of topologically nontrivial bundles is straightforward and not difficult.

Let us consider the $(0,2)$-component $F_{A}^{0,2}$ of the curvature tensor of a connection 1-form on $P$ and a $\mathfrak{g}$-valued $(n, n-2)$-form $B$ on $Z$. We introduce the action

$$
S_{\mathrm{hBF}}=\int_{Z} \operatorname{Tr}\left(B \wedge F_{A}^{0,2}\right)
$$

and models with such actions will be called holomorphic BF theories. The field equations obtained from the action (2.1) are

$$
\begin{gathered}
\bar{\partial} A^{0,1}+A^{0,1} \wedge A^{0,1}=0, \\
\bar{\partial} B+A^{0,1} \wedge B-B \wedge A^{0,1}=0 .
\end{gathered}
$$

Solutions $A^{0,1}$ of Eqs.(2.2a) will be called flat $(0,1)$-connections on $P$. Notice that Eqs.(2.2a) coincide with the compatibility conditions $\bar{\partial}_{A}^{2}=0$ of Eqs. $(2.2 \mathrm{~b})$. So, holomorphic BF theories describe flat $(0,1)$-connections on $P$ which correspond to holomorphic structures on the bundle $P \rightarrow Z$.

By expanding $A^{0,1}$ and $B$ in the generators $\left\{J^{i}\right\}$ of the group $G$, one can rewrite Eqs.(2.2) in the form

$$
\begin{gathered}
\bar{\partial} A^{i}+\frac{1}{2} f_{j k}^{i} A^{j} \wedge A^{k}=0, \\
\bar{\partial} B^{i}+f_{j k}^{i} A^{j} \wedge B^{k}=0,
\end{gathered}
$$

where $A^{0,1}=A^{i} J_{i}, B=B^{i} J_{i}$, and $f_{j k}^{i}$ are structure constants of the group $G, i, j, \ldots=1, \ldots, \operatorname{dim}_{\mathbb{C}} G$. Notice that Eqs.(2.3b) can be rewritten in the form $\bar{\partial}_{A} B=0$, where $\bar{\partial}_{A}=\bar{\partial}+\operatorname{ad} A^{0,1}$.

The gauge group $\mathcal{G}$ acts on a $(0,1)$-connection $A^{0,1}$ on $P$ and on a field $B \in \Omega^{n, n-2}(Z, \mathfrak{g})$ by the formulas

$$
\begin{gathered}
A^{0,1} \mapsto \operatorname{Ad}_{g^{-1}} A^{0,1}=g^{-1} A^{0,1} g+g^{-1} \bar{\partial} g, \\
B \mapsto \operatorname{Ad}_{g^{-1}} B=g^{-1} B g,
\end{gathered}
$$

where $g \in \mathcal{G}$. The action (2.1) is invariant under the transformations (2.4) and under the following "cohomological" symmetry transformation:

$$
B \mapsto B+\bar{\partial}_{A} \Phi
$$

where $\Phi \in \Omega^{n, n-3}(Z, \mathfrak{g})$. By virtue of this invariance, $\bar{\partial}_{A} \Phi$ is a trivial solution of Eqs.(2.2b) for any $\Phi \in \Omega^{n, n-3}(Z, \mathfrak{g})$, and solutions $B$ and $B+\bar{\partial}_{A} \Phi$ of Eqs.(2.2b) are considered as equivalent. 
We denote by $\mathcal{N}$ the space of solutions to Eqs.(2.2a) and by $\mathcal{M}$ the set of orbits of the gauge group $\mathcal{G}$ in the set $\mathcal{N}$,

$$
\mathcal{M}=\mathcal{N} / \mathcal{G}
$$

Let $\pi$ be a projection

$$
\pi: \mathcal{N} \rightarrow \mathcal{M}
$$

By definition, $\mathcal{N}$ is the space of flat $(0,1)$-connections (holomorphic structures) on the bundle $P$, and $\mathcal{M}$ is the moduli space of flat $(0,1)$-connections. Put another way, the moduli space $\mathcal{M}$ of holomorphic structures on $P$ is the space of gauge inequivalent solutions to Eqs.(2.2a).

Equations $(2.2 \mathrm{~b})$ are linear in $B$. For any fixed flat $(0,1)$-connection $A^{0,1}$ the space $\mathcal{B}_{A}$ of nontrivial solutions to Eqs. $(2.2 \mathrm{~b})$ is the $(n, n-2)$ th Dolbeault cohomology group

$$
\mathcal{B}_{A}=H_{\bar{\partial}_{A} ; P}^{n, n-2}(Z):=\frac{\left\{B \in \Omega^{n, n-2}(Z, \mathfrak{g}): \bar{\partial}_{A} B=0\right\}}{\left\{B=\bar{\partial}_{A} \Phi, \Phi \in \Omega^{n, n-3}(Z, \mathfrak{g})\right\}}
$$

which is defined to be the space of equivalence classes of $\bar{\partial}_{A}$-closed $\mathfrak{g}$-valued $(n, n-2)$-forms on $Z$, modulo $\bar{\partial}_{A}$-exact $\mathfrak{g}$-valued $(n, n-2)$-forms. So, the space of nontrivial solutions to Eqs. $(2.2 \mathrm{~b})$ forms the vector space $\mathcal{B}_{A}$ depending on a solution $A^{0,1}$ of Eqs.(2.2a). Therefore the space of solutions to Eqs.(2.2) forms a vector bundle $\mathcal{T} \rightarrow \mathcal{N}$, the base space of which is the space $\mathcal{N}$ of solutions to Eqs. (2.2a), and fibers of the bundle $\mathcal{T}$ at the points $A^{0,1} \in \mathcal{N}$ are the vector spaces $H_{\bar{\partial}_{A} ; P}^{n, n-2}(Z)=\mathcal{B}_{A}$ of nontrivial solutions to Eqs.(2.2b).

Recall that the gauge group $\mathcal{G}$ acts on solutions $\left(A^{0,1}, B\right)$ of Eqs.(2.2) by formulas (2.4). Therefore, identifying points $\left(A^{0,1}, B\right) \in \mathcal{T}$ and $\left(g^{-1} A^{0,1} g+g^{-1} \bar{\partial} g, g^{-1} B g\right) \in \mathcal{T}$ for any $g \in \mathcal{G}$, we obtain the moduli space

$$
\mathfrak{M}=\mathcal{T} / \mathcal{G}
$$

of solutions to Eqs.(2.2). The space $\mathfrak{M}$ is a vector bundle over the moduli space $\mathcal{M}$ of flat $(0,1)$ connections. If we denote by $\left[A^{0,1}\right]$ the gauge equivalence class of a flat $(0,1)$-connection $A^{0,1}$, then the fiber of this bundle at the point $\left[A^{0,1}\right] \in \mathcal{M}$ will be isomorphic to the Dolbeault cohomology group $H_{\bar{\partial}_{A} ; P}^{n, n-2}(Z)$. Thus, non-Abelian holomorphic BF theories give a field-theoretic description of holomorphic structures on bundles $P \rightarrow Z$ and of the twisted Dolbeault complex on $Z$.

\subsection{Abelian models}

In the Abelian case, instead of a $\mathfrak{g}$-valued $(0,1)$-connection on a bundle over a complex manifold $Z$ one can take any $(p, q-1)$-form $A^{p, q-1} \in \Omega^{p, q-1}(Z)$ and introduce the action

$$
S_{\mathrm{hAB}}=\int_{Z} B^{n-p, n-q} \wedge \bar{\partial} A^{p, q-1}
$$

where $B^{n-p, n-q} \in \Omega^{n-p, n-q}(Z)$, i.e. $B^{n-p, n-q}$ is a $\mathbb{C}$-valued $(n-p, n-q)$-form on $Z$. Equations of motion following from (2.9) are

$$
\begin{gathered}
\bar{\partial} A^{p, q-1}=0, \\
\bar{\partial} B^{n-p, n-q}=0 .
\end{gathered}
$$

The action (2.9) and Eqs.(2.10) are invariant under the following symmetry transformations:

$$
A^{p, q-1} \mapsto A^{p, q-1}+\bar{\partial} \Phi^{p, q-2},
$$




$$
B^{n-p, n-q} \mapsto B^{n-p, n-q}+\bar{\partial} \Phi^{n-p, n-q-1},
$$

where $\Phi^{r, s} \in \Omega^{r, s}(Z)$. Therefore the moduli space of solutions to Eqs.(2.10) is a vector space

$$
\mathcal{M}_{\mathrm{hAB}}=H_{\bar{\partial}}^{p, q-1}(Z) \oplus H_{\bar{\partial}}^{n-p, n-q}(Z)
$$

where $H_{\bar{\partial}}^{r, s}(Z)$ is the $(r, s)$ th Dolbeault cohomology group of $Z$. So, the action (2.9) provides us with a field-theoretic description of the standard Dolbeault complex on $Z$.

\section{Special holomorphic BF theories}

\subsection{Hermitian-Yang-Mills connections and stable bundles}

Let $Z$ be a Kähler $n$-manifold with a Kähler form $\omega$, and $G_{\mathbb{R}}$ be a subgroup of the unitary group $U(l)$ such that $\left(G_{\mathbb{R}}\right)^{\mathbb{C}}=G_{\mathbb{R}} \otimes \mathbb{C}=G, l \geq 2$. We consider a (trivial) principal $G_{\mathbb{R}}$-bundle $P_{\mathbb{R}}$ over $Z$ and the $(0,1)$-component $A^{0,1}$ of a unitary connection 1-form $A=A^{1,0}+A^{0,1}$ on $P_{\mathbb{R}}$.

Recall that any unitary connection $A$ on $P_{\mathbb{R}}$ defines a holomorphic structure on a $G$-bundle $P$ over $Z$ if the $(0,1)$-component $A^{0,1}$ of $A$ is flat, i.e. satisfies Eqs.(2.2a). Connections $A$ are called Hermitian-Yang-Mills connections, if their curvature $F_{A}$ satisfies the following equations [10]:

$$
\begin{gathered}
F_{A}^{0,2}=0, \\
\omega^{n-1} \wedge F_{A}^{1,1}=0,
\end{gathered}
$$

where

$$
\begin{gathered}
F_{A}^{0,2}=\bar{\partial} A^{0,1}+A^{0,1} \wedge A^{0,1} \\
F_{A}^{1,1}=\partial A^{0,1}+\bar{\partial} A^{1,0}+A^{1,0} \wedge A^{0,1}+A^{0,1} \wedge A^{1,0} .
\end{gathered}
$$

Hermitian-Yang-Mills connections correspond to stable holomorphic structures $\bar{\partial}_{A}$ on a bundle [10], but this is in some sense most of them.

Notice that Eqs.(3.1) can be rewritten in the form

$$
\begin{gathered}
F_{A}^{0,2}=0, \\
\Lambda F_{A}^{1,1}=0,
\end{gathered}
$$

where $\Lambda$ is an algebraic 'trace' operator which measures the component of a $(1,1)$-form parallel to $\omega$ [10]. In local coordinates $\left\{z^{a}\right\}$ on $Z$, Eqs.(3.3) have the form

$$
\begin{gathered}
F_{\bar{a} \bar{b}}=0, \\
g^{a \bar{b}} F_{a \bar{b}}=0,
\end{gathered}
$$

where $\mathbf{g}=\left\{g^{a \bar{b}}\right\}$ is a Riemannian metric compatible with the complex structure on $Z$. Equations (3.3) appear in superstring theory from the condition of preserving at least one unbroken supersymmetry in four dimensions after compactification of $D=10$ superstrings on Calabi-Yau 3 -folds [11].

The study of Eqs.(3.1) or equivalent Eqs.(3.3) is of interest because of their importance in algebraic geometry and superstring theory. We introduce an action that leads to equations of motion containing Eqs.(3.1). Namely, consider the action

$$
S_{\mathrm{HYM}}=S_{\mathrm{hBF}}+\beta S_{\omega \Xi \mathrm{F}}=\int_{Z} \operatorname{Tr}\left(B \wedge F_{A}^{0,2}-\beta \Xi \omega^{n-1} \wedge F_{A}^{1,1}\right),
$$


where $\Xi$ is a smooth $\mathfrak{g}$-valued function on $Z$ and $\beta$ is a constant parameter. The action (3.5) is a one-parameter deformation of the action $S_{\mathrm{hBF}}$ for holomorphic BF theories introduced in Sect.2.1. Let us emphasize that the action $S_{\mathrm{HYM}}$ depends on a Kähler form $\omega$ on $Z$.

The field equations following from the action (3.5) are

$$
\begin{gathered}
F_{A}^{0,2}=0, \quad \beta \omega^{n-1} \wedge F_{A}^{1,1}=0, \\
\bar{\partial} B+A^{0,1} \wedge B-B \wedge A^{0,1}=\partial\left(\beta \Xi \omega^{n-1}\right)+\omega^{n-1} \wedge\left[A^{1,0}, \beta \Xi\right], \\
\bar{\partial}\left(\beta \Xi \omega^{n-1}\right)+\omega^{n-1} \wedge\left[A^{0,1}, \beta \Xi\right]=0,
\end{gathered}
$$

where $[$,$] is the standard commutator in the Lie algebra \mathfrak{g}$. We see that Eqs.(3.6a) coincide with Eqs.(3.1) and therefore $A=A^{1,0}+A^{0,1}$ is a Hermitian-Yang-Mills connection. It is obvious that if $\beta=0$, then Eqs.(3.6) coincide with Eqs.(2.2) of a holomorphic BF theory.

\subsection{Holomorphic $\theta \mathrm{BF}$ theories on Calabi-Yau manifolds}

Let now $Z$ be a Calabi-Yau $n$-fold. This means that besides a complex structure, on $Z$ there exist a Kähler 2-form $\omega$, a Ricci-flat Kähler metric $\mathbf{g}$ and a nowhere vanishing holomorphic $(n, 0)$-form $\theta$. The $(n, 0)$-form $\theta$ can be taken to be covariantly constant w.r.t. the Levi-Civita connection. We consider a (trivial) principal $G$-bundle $P$ over $Z$ and the $(0,1)$-component $A^{0,1}$ of a connection 1-form $A$ on $P$.

For bundles $P$ over Calabi-Yau (CY) $n$-folds $Z$ one can consider holomorphic BF theories with the action (2.1) which does not depend on Kähler structures, metrics and holomorphic $(n, 0)$-forms on $Z$. But the existence on $Z$ of a nowhere degenerate holomorphic $(n, 0)$-form $\theta$ permits one to introduce one more class of models which will be called holomorphic $\theta B F$ theories. Their action has the form

$$
S_{\mathrm{h} \theta \mathrm{BF}}=\int_{Z} \theta \wedge \operatorname{Tr}\left(B \wedge F_{A}^{0,2}\right),
$$

where $B$ is a $\mathfrak{g}$-valued $(0, n-2)$-form on $Z$, and $F_{A}^{0,2}=\bar{\partial} A^{0,1}+A^{0,1} \wedge A^{0,1}$ is the (0,2)-component of the curvature tensor of a connection $A$ on $P$. The action (3.7) leads to the equations of motion

$$
\theta \wedge F_{A}^{0,2}=0, \quad \theta \wedge\left(\bar{\partial} B+A^{0,1} \wedge B-(-1)^{n} B \wedge A^{0,1}\right)=0
$$

which are equivalent to the equations

$$
\begin{gathered}
\bar{\partial} A^{0,1}+A^{0,1} \wedge A^{0,1}=0 \\
\bar{\partial} B+A^{0,1} \wedge B-(-1)^{n} B \wedge A^{0,1}=0 .
\end{gathered}
$$

In abridged notation, Eqs.(3.9b) can be rewritten as $\bar{\partial}_{A} B=0$.

From Eqs.(3.9) we see that holomorphic $\theta$ BF theories describe holomorphic structures on bundles $P \rightarrow Z$ and $\bar{\partial}_{A}$-closed forms $B \in \Omega^{0, n-2}(Z, \mathfrak{g})$. Solutions to Eqs.(3.9a) are flat $(0,1)$-connections and for any fixed flat $(0,1)$-connection $A^{0,1}$ on $P$ the space of nontrivial solutions to Eqs.(3.9b) is the $(0, n-2)$ th Dolbeault cohomology group

$$
H_{\bar{\partial}_{A} ; P}^{0, n-2}:=\frac{\left\{B \in \Omega^{0, n-2}(Z, \mathfrak{g}): \bar{\partial}_{A} B=0\right\}}{\left\{B=\bar{\partial}_{A} \Phi, \Phi \in \Omega^{0, n-3}(Z, \mathfrak{g})\right\}} .
$$

So, the action (3.7) provides us with a field-theoretic description of the Dolbeault complex coupled to a flat $(0,1)$-connection on the adjoint bundle ad $P$ over a Calabi-Yau $n$-fold $Z$. 


\subsection{Special hBF theories on twistor spaces of self-dual 4-manifolds}

Let us consider a Riemannian real 4-manifold $M$ with self-dual Weyl tensor (a self-dual manifold) and the bundle $\tau: Z \rightarrow M$ of complex structures on $M$ (the twistor space of $M$ ) with $\mathbb{C} P^{1}$ as a typical fiber [12, 13]. The twistor space $Z$ of a self-dual 4-manifold $M$ is a complex 3-manifold [13] which is the total space of a fiber bundle over $M$ associated with the bundle of orthonormal frames on $M$.

It is well known that Yang-Mills instantons on $M$ can be described in terms of holomorphic bundles over the twistor space $Z$ of $M$. Namely, there is a one-to-one correspondence between selfdual bundles (bundles with self-dual connections) over $M$ and holomorphic bundles $P$ over $Z$ that are holomorphically trivial on any projective line $\mathbb{C} P_{x}^{1} \hookrightarrow Z$ parametrized by $x \in M$ [14, 15, 13]. The condition of holomorphic trivializability of the bundle $P$ after the restriction of $P$ to every projective line in $Z$ is equivalent to the equality to zero of the restriction of the $(0,1)$-component $A^{0,1}$ of a connection $A$ on $P$ to submanifolds $\mathbb{C} P_{x}^{1} \hookrightarrow Z, x \in M$ (see [16] for discussions). Such connections on $P$ can be described in terms of holomorphic BF theories on $Z$ by adding a term to the action (2.1). In this way we obtain a model which describes the instanton moduli space and differs from the Donaldson-Witten model.

Consider the twistor space $Z$ of a self-dual real 4-manifold $M$. By definition, $Z$ is a complex fibered 3-manifold with the canonical projection $\tau: Z \rightarrow M$. The typical fiber $\mathbb{C} P^{1}$ has the $S U(2)$ invariant complex structure (see [17] for more details), and the vertical distribution $\mathcal{V}=\operatorname{Ker} \tau_{*}$ inherits this complex structure. A restriction of $\mathcal{V}$ to each fiber $\mathbb{C} P_{x}^{1}, x \in M$, is the tangent bundle to that fiber. The Levi-Civita connection on the Riemannian manifold $M$ generates the splitting of the tangent bundle $T(Z)$ into a direct sum

$$
T(Z)=\mathcal{V} \oplus \mathcal{H}
$$

of the vertical $\mathcal{V}$ and horizontal $\mathcal{H}$ distributions. Using the complex structures on $\mathbb{C} P^{1}$ and $Z$, one can split the complexified tangent bundle of $Z$ into a direct sum

$$
T^{\mathbb{C}}(Z)=T^{1,0} \oplus T^{0,1}=\left(\mathcal{V}^{1,0} \oplus \mathcal{H}^{1,0}\right) \oplus\left(\mathcal{V}^{0,1} \oplus \mathcal{H}^{0,1}\right)
$$

of subbundles of type $(1,0)$ and $(0,1)$. Analogously one can split the complexified cotangent bundle of $Z$ into a direct sum of subbundles $T_{1,0}$ and $T_{0,1}$. So we have the (integrable) distribution $\mathcal{V}^{0,1}$ of $(0,1)$-vector fields.

Let $E^{3,3}$ be a $\mathfrak{g}$-valued $(3,3)$-form on $Z$, and $V^{0,1}$ be an arbitrary $(0,1)$-vector field from the distribution $\mathcal{V}^{0,1}$. Denote by $\left\{e_{a}\right\},\left\{e_{\bar{a}}\right\},\left\{\sigma^{a}\right\}$ and $\left\{\sigma^{\bar{a}}\right\}$ local frames for the bundles $T^{1,0}, T^{0,1}, T_{1,0}$ and $T_{0,1}$, respectively. Then locally

$$
E^{3,3}=E_{a_{1} \ldots \bar{a}_{3}} \sigma^{a_{1}} \wedge \ldots \wedge \sigma^{\bar{a}_{3}}, \quad V^{0,1}=V^{\bar{a}} e_{\bar{a}}, \quad A^{0,1}=A_{\bar{a}}^{0,1} \sigma^{\bar{a}},
$$

where $a_{1}, \bar{a}_{1}, \ldots=1,2,3$. Let us consider the contraction

$$
\left.V^{0,1}\right\lrcorner E^{3,3}
$$

of $V^{0,1}$ with $E^{3,3}$, which is a $\mathfrak{g}$-valued $(3,2)$-form on $Z$. In the local frames, the form (3.14a) has components

$$
\left.\left(V^{0,1}\right\lrcorner E^{3,3}\right)_{a_{1} a_{2} a_{3} \bar{a}_{1} \bar{a}_{2}}:=3 V^{\bar{a}_{3}} E_{a_{1} a_{2} a_{3} \bar{a}_{1} \bar{a}_{2} \bar{a}_{3}} .
$$

We also introduce a $\mathfrak{g}$-valued function

$$
\left.V^{0,1}\right\lrcorner A^{0,1}=V^{\bar{a}} A_{\bar{a}}^{0,1}
$$


on $Z$ and notice that

$$
\left.\left.\left(V^{0,1}\right\lrcorner E^{3,3}\right) \wedge A^{0,1}=-E^{3,3}\left(V^{0,1}\right\lrcorner A^{0,1}\right)
$$

since $E^{3,3} \wedge A^{0,1} \equiv 0$.

To the action (2.1) of a holomorphic BF theory we add the term

$$
\left.S_{\mathrm{VAE}}=\gamma \int_{Z} \operatorname{Tr}\left[\left(V^{0,1}\right\lrcorner E^{3,3}\right) \wedge A^{0,1}\right]
$$

where $\gamma=$ const. The action

$$
\left.S_{\mathrm{hBF}}+S_{\mathrm{VAE}}=\int_{Z} \operatorname{Tr}\left[B^{3,1} \wedge F_{A}^{0,2}-\gamma\left(V^{0,1}\right\lrcorner A^{0,1}\right) E^{3,3}\right]
$$

leads to the following field equations:

$$
\begin{gathered}
\left.\bar{\partial} A^{0,1}+A^{0,1} \wedge A^{0,1}=0, \quad \gamma V^{0,1}\right\lrcorner A^{0,1}=0, \\
\left.\bar{\partial} B^{3,1}+A^{0,1} \wedge B^{3,1}-B^{3,1} \wedge A^{0,1}=\gamma V^{0,1}\right\lrcorner E^{3,3} .
\end{gathered}
$$

Equations (3.18a) on the twistor space $Z$ of a self-dual 4-manifold $M$ are equivalent to the self-dual Yang-Mills (SDYM) equations on $M$, which was discussed in detail in [16, 17]. Equations (3.18b) describe some fields interacting with external self-dual gauge fields on $M$. It is obvious that in the limit $\gamma \rightarrow 0$, Eqs.(3.18) are reduced to Eqs.(2.2) of holomorphic BF theories on $Z$.

\subsection{Conformal and integrable field theories related to special hBF theories}

It is well known that Chern-Simons theories on $3 D$ real manifolds $X$ are reduced to $2 D$ conformal field theories (CFT) if one supposed that a 3-manifold $X$ has the form of a trivial bundle $\Sigma \times$ $\mathbb{R}\left(\right.$ or $\Sigma \times S^{1}$ ), where $\Sigma$ is a 2-manifold [2, 18]. It is reasonable to expect that holomorphic analogs of Chern-Simons and BF theories defined on a $3 D$ complex manifold $Z$ (six real dimensions) can be reduced to $4 D$ CFT's if one supposes that $Z$ is a bundle over a real 4-manifold $M$ with two-dimensional fibers. One important example of a conformal field theory in four dimensions is provided by the SDYM model. In Sect.3.3 we have described the reduction of holomorphic BF models on $Z$ to SDYM models on self-dual 4-manifolds $M$. For discussions of reductions to other $4 D$ CFT's, see [16].

Notice that Hermitian-Yang-Mills connections (see Sect.3.1) on bundles $P$ over complex 2dimensional Kähler manifolds are self-dual connections since Eqs.(3.6a) for $n=2$ coincide with the SDYM equations. Thus, both the action (3.5) with $n=2$ and the action (3.17) describe SDYM models on 4-manifolds. In the simplest case of the flat 4-space, we obtain SDYM models on $\mathbb{R}^{4}$. It is well known that almost all integrable equations in $1 \leq D \leq 3$ dimensions (Bogomolny, Korteweg-de Vries, Nonlinear Schrödinger, Boussinesq, Nahm and many others) can be obtained by reductions of the SDYM equations on $\mathbb{R}^{4}$ (see [19, 20] and references therein). Thus, special holomorphic BF theories are connected with $4 D$ CFT's and integrable models in $1 \leq D \leq 4$ dimensions. 


\section{Observables and topological invariants}

To introduce observables and topological invariants for holomorphic $\mathrm{BF}$ and $\theta \mathrm{BF}$ theories we use the results on holomorphic analogs of Chern-Simons theories defined on Calabi-Yau 3-folds [6, 7, 8, 9].

First, for an action $S$ coinciding with $(2.1)$, (2.9) or (3.7), one can write down the partition function

$$
Z_{k}=\int_{\mathcal{A} / \mathcal{G}} \mathcal{D} A^{0,1} \exp (i k S),
$$

where $k \in \mathbb{Z}$, and the path integral is evaluated over the space $\mathcal{A} / \mathcal{G}$ of gauge inequivalent $(0,1)$ connections $A^{0,1}$ on a bundle $P \rightarrow Z$.

Second, using the semiclassical approximation, one can give the partition function interpretation of the Ray-Singer holomorphic torsion [21] of the holomorphic bundle $\left(\operatorname{ad} P, \bar{\partial}_{A}\right)$.

Third, one can introduce an analog of Wilson loops for Abelian $(0,1)$-connections $A^{0,1}$ on a line bundle $L \rightarrow Z$ [9]. For this one should fix complex curves $C_{i} \subset Z$ (e.g. tori), holomorphic 1-forms $\alpha_{i}$ on the $C_{i}$ 's and define

$$
T\left(C_{i}\right)=\exp \left(i k \int_{C_{i}} \alpha_{i} \wedge A^{0,1}\right)
$$

as an element of the Jacobian of $L$ paired against $\alpha_{i}$ and exponentiated. Then we can consider the path integral

$$
\int_{\mathcal{A} / \mathcal{G}} \mathcal{D} A^{0,1} \exp (i k S) \prod_{i=1}^{m} T\left(C_{i}\right)
$$

and try to prove that it is an invariant of the complex structure of a manifold $Z$.

Finally, for Abelian holomorphic BF theories with the action (2.9) one can introduce the Dolbeault currents 22] and define the linking number of complex submanifolds of a manifold $Z$ using a pairing $H_{\bar{\partial}}^{p, q}(Z) \otimes H_{\bar{\partial}}^{n-p, n-q}(Z) \rightarrow \mathbb{C}$ of the Dolbeault cohomology groups.

\section{Conclusion}

In this paper we have introduced new classes of gauge field theories which are natural holomorphic analogs of BF topological theories. These holomorphic BF theories give a field-theoretic description of holomorphic structures on bundles over complex $n$-manifolds. Three special holomorphic BF theories on Kähler, Calabi-Yau and fibered complex manifolds have been introduced. In particular, we have introduced an action describing Hermitian-Yang-Mills connections on stable holomorphic bundles. Such connections satisfy the Donaldson-Uhlenbeck-Yau equations and are often used in superstring theory in the description of compactified configurations with unbroken supersymmetry in four dimensions.

There are many open problems that have to be considered. In particular, it is necessary to analyze the ghost structure of the introduced theories, to perform BRST gauge fixing and write down quantum actions. Supersymmetrization of holomorphic BF theories may be of interest. The problem of finding nontrivial observables and their metric independence should be more carefully analyzed. Much work remains to be done. 


\section{References}

[1] E.Witten, Commun. Math. Phys. 117 (1988) 353.

[2] E.Witten, Commun. Math. Phys. 121 (1989) 351.

[3] D.Birmingham, M.Blau, M.Rakowski and G.Thompson, Phys.Rep. 209 (1991) 129;

J.M.F.Labastida and C.Losano, Lectures on topological quantum field theory, In: Proc. La Plata-CERN-Santiago de Compostela meeting on Trends in theoretical physics (La Plata 1997), eds. H.Falomir, R.Gamboa and F.Schaposnik (AIP, New York, 1998) p.54, hepth/9709192.

[4] G.T.Horowitz, Commun. Math. Phys. 125 (1989) 417;

M.Blau and G.Thompson, Ann.Phys. 205 (1991) 130.

[5] A.S.Schwarz, Lett.Math.Phys. 2 (1978) 247.

[6] E.Witten, Chern-Simons gauge theory as a string theory, In: The Floer Memorial Volume, Progr. Math. 133 (Birkhäuser, Boston, 1995) p.637, hep-th/9207094.

[7] M.Bershadsky, S.Cecotti, H.Ooguri and C.Vafa, Commun. Math. Phys. 165 (1994) 311, hepth/9309140.

[8] S.K.Donaldson and R.P.Thomas, Gauge theory in higher dimensions, In: The Geometric Universe, Oxford 1996 (Oxford University Press, 1998) p.31.

[9] R.P.Thomas, Gauge theory on Calabi-Yau manifolds, D.Phil.Thesis, University of Oxford, 1997.

[10] S.K.Donaldson, Proc. London Math. Soc. 50 (1985) 1;

K.Uhlenbeck and S.-T.Yau, Commun. Pure Appl. Math. 39 (1986) 257.

[11] M.B.Green, J.H.Schwarz and E.Witten, Superstring theory, Vol.2 (Cambridge University Press, Cambridge, 1988).

[12] R.Penrose, Gen. Rel. Grav. 7 (1976) 31.

[13] M.F.Atiyah, N.J.Hitchin and I.M.Singer, Proc. R. Soc. Lond. A362 (1978) 425.

[14] R.S.Ward, Phys.Lett. 61A (1977) 81.

[15] M.F.Atiyah and R.S.Ward, Commun. Math. Phys. 55 (1977) 117;

M.F.Atiyah, V.G. Drinfeld, N.J.Hitchin and Yu.I.Manin, Phys. Lett. 65A (1978) 185.

[16] A.D.Popov, Nucl.Phys. B550 (1999) 585, hep-th/9806239.

[17] A.D.Popov, Rev. Math. Phys. 11 (1999) 1091, hep-th/9803183.

[18] G.Moore and N.Seiberg, Phys. Lett. B220 (1989) 422;

S.Elitzur, G.Moore, A.Schwimmer and N.Seiberg, Nucl. Phys. B326 (1989) 108.

[19] R.S.Ward, Phil.Trans.R.Soc.Lond. A315 (1985) 451;

L.J.Mason and G.A.J.Sparling, Phys.Lett. A137 (1989) 29;

T.A. Ivanova and A.D. Popov, Theor.Math.Phys. 102 (1995) 280;

M.Legaré, Int.J.Mod.Phys. A12 (1997) 219. 
[20] L.J.Mason and N.M.J.Woodhouse, Integrability, self-duality and twistor theory (Oxford University Press, Oxford, 1996).

[21] D.Ray and I.Singer, Ann.Math. 98 (1973) 154.

[22] P.Griffiths and J.Harris, Principles of algebraic geometry (Wiley \& Sons, New York, 1978). 